[32] P. C. Arnett and B. H. Yun, "Silicon nitride trap properties as revealed by charge centroid measurements on MNOS devices," Appl. Phys. Lett., vol. 96, pp. 94-96, Feb. 1975.

[33] R. R. Troutman and S. N. Chakravarti, "Subthreshold characteristics of insulated gate field-effect transistors," IEEE Trans. Circuit Theory, vol. CT-20, pp. 659-665, 1973.

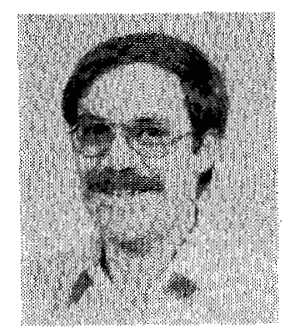

Peter E. Cottrell ( $\left.{ }^{\prime} 69-M^{\prime} 73\right)$ was born in Troy, N.Y. He received the B.S., M.E. and Ph.D. degrees from Rensselaer Polytechnic Institute, Troy, NY, in 1968, 1970, and 1973, respectively.

From 1970 to 1972 , he was an Instructor at Rensselaer Polytechnic Institute. Since 1973, he has been employed by the IBM General Technology Division, Essex Junction, VT. His experience has included research and development in the following areas: transient ionizing radiation effects in IMPATT diodes, hot-electron effects in MOSFET's, design limitations and characterization of MOSFET structures, and twodimensional simulation of both bipolar and FET semiconductor devices.

Dr. Cottrell is a member of Eta Kappa Nu, Tau Beta Pi, and Sigma Xi.

Ronald R. Troutman (S'61-M'63-SM'78), for a biography and photograph, see this issue, p. 469.

T. H. Ning (M'75), for a biography and photograph see this issue, p. 352.

\title{
Cost and Performance of VLSI Computing Structures
}

\author{
CARVER A. MEAD AND MARTIN REM
}

\begin{abstract}
Using VLSI technology, it will soon be possible to implement entire computing systems on one monolithic silicon chip. Conducting paths are required for communicating information throughout any integrated system. The length and organization of these communication paths place a lower bound on the area and time required for system operations. Optimal designs can be achieved in only a few of the many alternative structures. Two illustrative systems are analyzed in detail: a RAM-based system and an associative system. It is shown that in each case an optimum design is possible using the area-time product as a cost function.
\end{abstract}

\section{INTRODUCTION}

$\mathbf{T}$ HE SILICON integrated-circuit technology is evolving continuously toward smaller elementary devices and denser, more complex functions on each single silicon chip. It appears that new processing and lithographic techniques will make possible the fabrication of chips containing $10^{7}$ or $10^{8}$ individual transistors. One such chip will contain more function than today's largest computers. A large amount of effort has been put into fabrication questions, and much more effort will be required to reach the practical limits of device

Manuscript received September 18, 1978; revised January 10, 1979. This work was supported in part by BMD under Contract DASG60-77C-0097, and the Office of Naval Research under Contract N00014-16C-0367. (California Institute of Technology, Computer Science Department Contribution 1584.)

C. A. Mead is with the Department of Computer Science, California Institute of Technology, Pasadena, CA 91125.

M. Rem is with the Department of Computer Science, California Institute of Technology, Pasadena, CA 91125, on leave from the Department of Mathematics, Eindhoven University of Technology, Eindhover, The Netherlands. compactness. However, there is at present essentially no theoretical basis for optimizing the overall organization of systems implemented in this technology.

The conventional complexity theory is inadequate because its measure of cost is the number of steps of a sequential machine. No account is taken of the size of the machine (and hence the time required for each step). Possible concurrency is ignored, thereby ruling out the most important potential contribution of the silicon technology. The traditional switching theory is also inadequate. While it provides a beautiful formalism for describing elementary logic functions, its optimization methods concern themselves with logical operations rather than communication requirements. Even in current integrated circuits, the wires required for communicating information across the chip account for most of the area, and driving these wires accounts for most of the time delay. In very large scale integrated systems, the situation becomes even more extreme. In this paper, we describe a method by which the conceptual organization of a large chip can be analyzed, and a lower bound placed on its size and cycle time before a detailed design is undertaken. The results of this analysis suggest rather general guidelines for the organization of large integrated systems.

\section{Metrics of Space ANd Time}

\section{A. Physical Properties}

Devices used to construct monolithic silicon integrated circuits are universally of the charge-controlled type. A charge $Q$ placed on the control electrode (gate, base, etc.) results in 
a current $I=Q / \tau$ flowing through the device. The transit time $\tau$ is the time required for charge carriers to move through the active region of the device.

All times in an integrated system can be formulated as simple multiples of $\tau$. For one transistor to drive another identical to it, a charge $Q$ must flow through its active region, requiring time $\tau$. If the capacitance $C_{L}$ of the load being driven is $K$ times the gate capacitance $C_{g}$ of the driving transistor, al time $K \tau=\left(C_{L} / C_{g}\right) \tau$ is required.

\section{B. Linear Versus Hierarchical Structures}

In large integrated systems it is necessary to commulicate information throughout the entire system. As an example, a bit of information stored on the gate of a minimum size transistor in a random-access memory must be communicated to the memory bus of a CPU. Since there are many words of data in the memory, there are many possible sources for each wire in the memory bus. Fig. 1 illustrates two possible approaches to organizing such a bus. In the first approach, a transistor associated with each bit drives the bus wire directly. If the bus wire has a capacitance $C_{w}$, the time required to drive the bus wire is $t=\tau\left(C_{w} / C_{g}\right)$. In a typical computer memory, $C_{w}$ is many orders of magnitude larger than $C_{g}$, and the delay introduced by such a scheme is very long. Sincs $C_{w}$ is proportional to the length of the wire, it is also proport onal to $S$, the number of driver transistors connected to the wise.

$$
t=\tau S \text {. }
$$

A second scheme is shown in Fig. 1(b). Here each transistor drives a wire only long enough to reach its neighbor. Each such wire is connected to the gate of a transistor twice as arge as the transistor driving it. The arrangement is repeatec upward until the top level where all sources have a path tc the bus. In this scheme the delay in driving the lowest level wire is $2 \tau$ (assuming the primary capacitance is due to the gate of the larger transistor). The delay introduced by the wirts at each level is the same, since each driver transistor is twice as large as those driving it. Hence the delay in driving the bus line is $2 \tau N$ where $N$ is the number of levels in the structure. Since there are $S=2^{N}$ transistors at the lowest level, the delay may be written

$$
t=2 \tau \log _{2} S
$$

Comparing (2) and (1), we see that for large $S$ the delay has been made much shorter by using a hierarchical structure.

\section{A Cost Criterion}

A hierarchy such as that shown in Fig. 1(b) may be built using any integral number $\alpha$ of transistors driving each wire. The driver transistors will in general be $\alpha$ times the ize of those driving them. The delay for such a structure is $t=$ $\alpha \tau \log _{\alpha} S=\tau(\alpha / \log \alpha) \log S$. All system delays are thus proprortional to $\tau \log S$, with a penalty factor $\alpha / \log \alpha$ dependent upon the branching ratio of the hierarchy. This delay is plottec in Fig. 2, normalized to its minimum value which is attainec at $\alpha=e$.

While dramatic improvements in the performance of ir.tegrated structures can be achieved by a hierarchical organization,

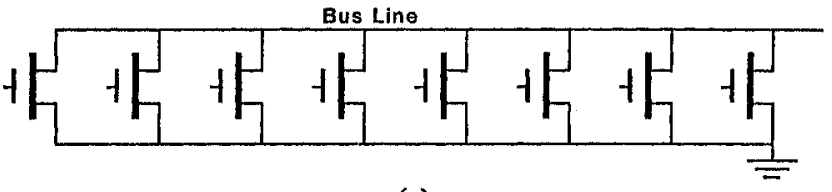

(a)

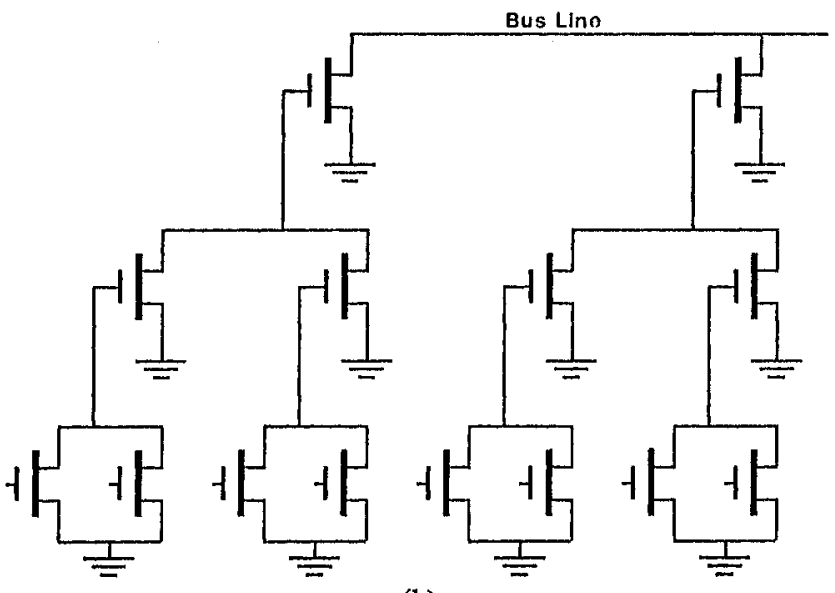

(b)

Fig. 1. (a) A bus driven directly by memory cells. (b) A bus driver tree.

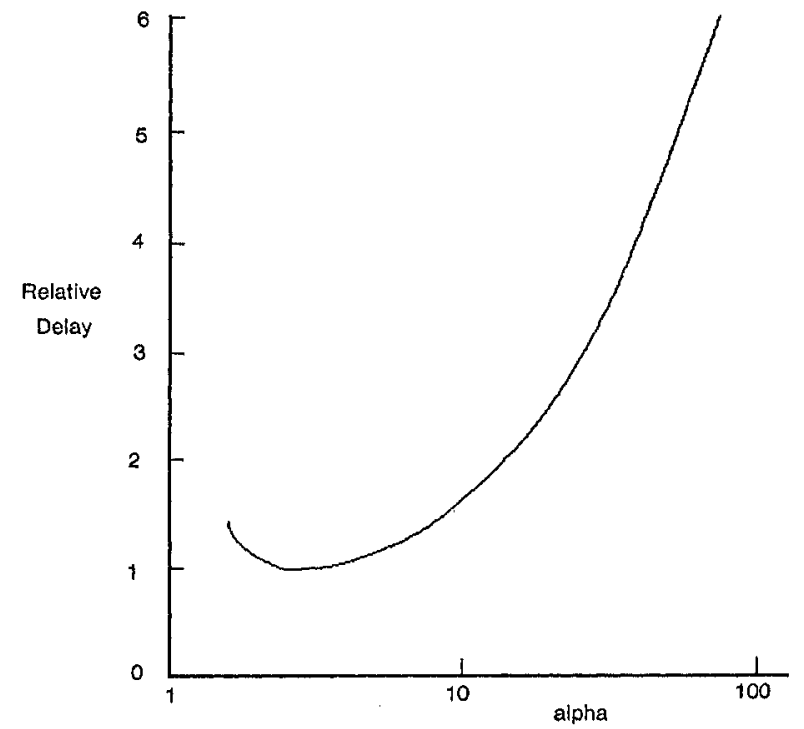

Fig. 2. Delay of a hierarchical structure as a function of $\alpha$.

a penalty is always paid in the area required for wires. In the simple case shown, a bus requiring one wire when driven directly requires $\log _{\alpha} S$ wires when organized as a hierarchy. For this reason it is not possible to optimize a design without a cost function involving both area and time. In this paper we will use the area-time product as our basic cost function. For the above simple example, the cost function is area time $=$ $\tau(\log S)^{2} \alpha /(\log \alpha)^{2}$. The cost is minimized for $\alpha=e^{2} \approx 7.4$.

\section{Hierarchical Computing Systems}

The analysis given above suggests a very general structure for computing systems. Lowest level cells are grouped together into modules in such a way that $\alpha$ cells drive their outputs onto an output wire. Each output wire is connected to a driver 
transistor which is $\alpha$ times as large as those driving the wire. Modules are grouped in such a way that $\alpha$ of those modules drivers are connected to an intermodule communication wire. This wire in turn is connected to a driver transistor $\alpha^{2}$ times as large as the lowest level transistors. This process is continued until the appropriate size system has been realized.

\section{RANDOM-ACCESS MEMORY}

In this section we discuss the cost and performance of a random-access memory (RAM) of $S$ words of $\log S$ bits each. As the unit of length we employ the minimum distance of two conducting paths. For the unit of time we choose the time it takes a basic element to charge a wire of unit length plus another transistor like itself. One unit of time is thus slightly larger than the transit time of a transistor.

\section{A. Organization of the $R A M$}

We organize the RAM in a hierarchical fashion. The elements of level 0 are the bits themselves, each bit consisting of two crossing wires: a select wire and a data wire. When the select wire is signaled it puts its contents on the data wire. We group $\alpha^{2}$ bits into an $\alpha \times \alpha$ square to form a module of level 1. If the width of an element (a bit) is $b_{0}$, the elements have to drive wires of length $\alpha b_{0}$. A module on level 1 consists of an array of crossing select and data wires, constituting the $\alpha^{2}$ bits of level 0 , and some additional logic and wires at the side. We group again $\alpha^{2}$ of these modules into a square to form a module of level 2, etc. Fig. 3 shows three levels of the hierarchy for $\alpha=4$.

To study the memory in more detail we look at a module of level $i$ (Fig. 4). We describe how one extracts one of its $\alpha^{2 i}$ bits. In order to select 1 bit of storage, $2 i \log \alpha$ address wires are required. We run $i \log \alpha$ of them, called the row address wires, vertically along the side of the module and the other $i \log \alpha$; the column address wires, horizontally. Its $\alpha^{2}$ submodules are organized into $\alpha$ rows of $\alpha$ submodules each. When the select wire of the module is asserted $\log \alpha$ of the row address wires are used by the decoder to select one of the $\alpha$ rows of submodules; the select wire running through that row is asserted. The other $(i-1) \log \alpha$ row address wires are run horizontally into each of the $\alpha$ rows of submodules, where they serve as column address wires for the submodules. Of the $i \log \alpha$ column address wires $(i-1) \log \alpha$ are run vertically into each of the $\alpha$ columns of submodules, where they serve as row addresses. The other $\log \alpha$ address wires are used by the multiplexor to select one of the $\alpha$ data wires coming out of the columns of submodules. The signal on the selected data wire is driven onto the data wire of the module itself.

If we wish to have a memory of $S$ words with $N+1$ levels (level 0 through $N$ ) we choose $N=\log S / 2 \log \alpha$ or $S=\alpha^{2 N}$. This gives a hierarchical structure with $S$ bits from which we can extract 1 bit at a time. If we want the word length to be $\log S$ we employ $\log S$ of these structures in parallel. To select one word we select 1 bit in each of the $\log S$ hierarchies.

\section{B. Area of the RAM}

Fig. 4 allows us to compute the size of a RAM. Let $L_{i}$ denote the width of a module of level $i$; then we have the following

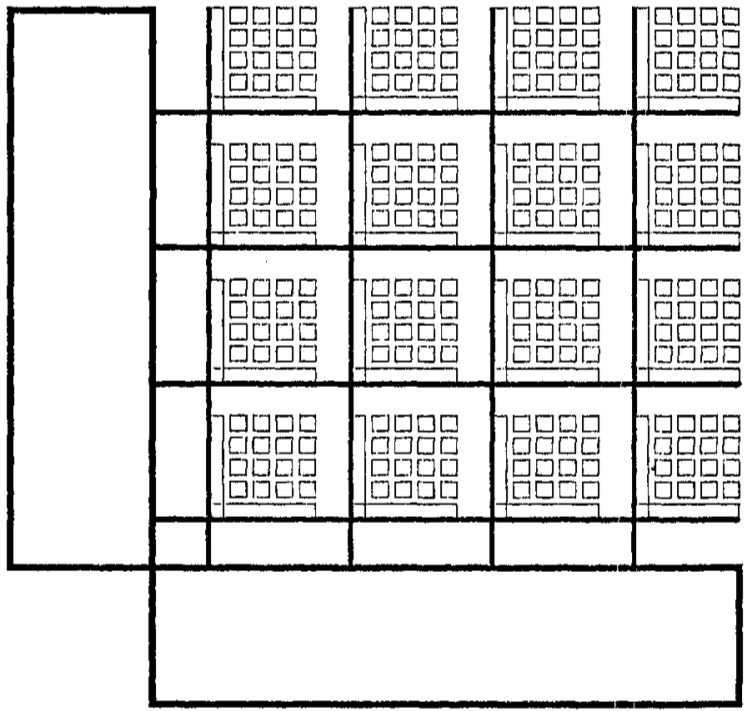

Fig. 3. Three levels of a memory hierarchy for $\alpha=4$.

recurrence relation:

$$
\begin{aligned}
& L_{0}=b_{0} \\
& L_{i}=i \log \alpha+1+\log \alpha+\alpha \cdot L_{i-1} .
\end{aligned}
$$

The solution to the above relation is

$$
L_{i}=\alpha^{i} b_{0}+\frac{\alpha^{i}-1}{\alpha-1}+\left(\frac{2 \alpha^{i+1}-\alpha^{i}-\alpha}{(\alpha-1)^{2}}-\frac{i+1}{\alpha-1}\right) \log \alpha .
$$

Rather than the width itself we are interested in the width per bit. In one direction, horizontal or vertical, module $i$ has $\alpha^{i}$ bits; therefore, we compute $L_{i} / \alpha^{i}$.

$$
\begin{aligned}
\frac{L_{i}}{\alpha^{i}}= & b_{0}+\frac{1}{\alpha-1}+\frac{2 \alpha-1}{(\alpha-1)^{2}} \log \alpha \\
& -\frac{1}{(\alpha-1) \alpha^{i}}\left[\left(\frac{\alpha}{\alpha-1}+1+i\right) \log \alpha+1\right] .
\end{aligned}
$$

An interesting property of the width per bit, as expressed by (3), is that its limit for $i \rightarrow \infty$ is finite.

$$
\lim _{i \rightarrow \infty} \frac{L_{i}}{\alpha^{i}}=b_{0}+\frac{1}{\alpha-1}+\frac{2 \alpha-1}{(\alpha-1)^{2}} \log \alpha .
$$

This means that the width per bit $L_{i} / \alpha^{i}$ is bounded from above by (4) independent of the number of levels of a RAM. Expression (3) converges in an exponential fashion towards its limit. For small values of $i,(3)$ is already very close to (4). Therefore, we use (4) as the width per bit for a RAM; its square is then the area per bit. By dividing the area per bit by the bit area $b_{0}^{2}$ we obtain the total area per bit area for a RAM. Fig. 5 shows this quotient as a function of $\alpha$ for four different values of $b_{0}$. It gives the overhead factor in the area that is due to the wires. For a memory of $64 \mathrm{~K}$ bits with $N=2, \alpha$ should be 16. Expression (4) is then equal to $b_{0}+0.6$. This shows that in 2-level $64 \mathrm{~K}$ dynamic MOS mernories, for which $b_{0}$ lies between 1 and 2 , roughly half of the area will be occupied by wires.

One may wonder why we have not discussed the area that is 


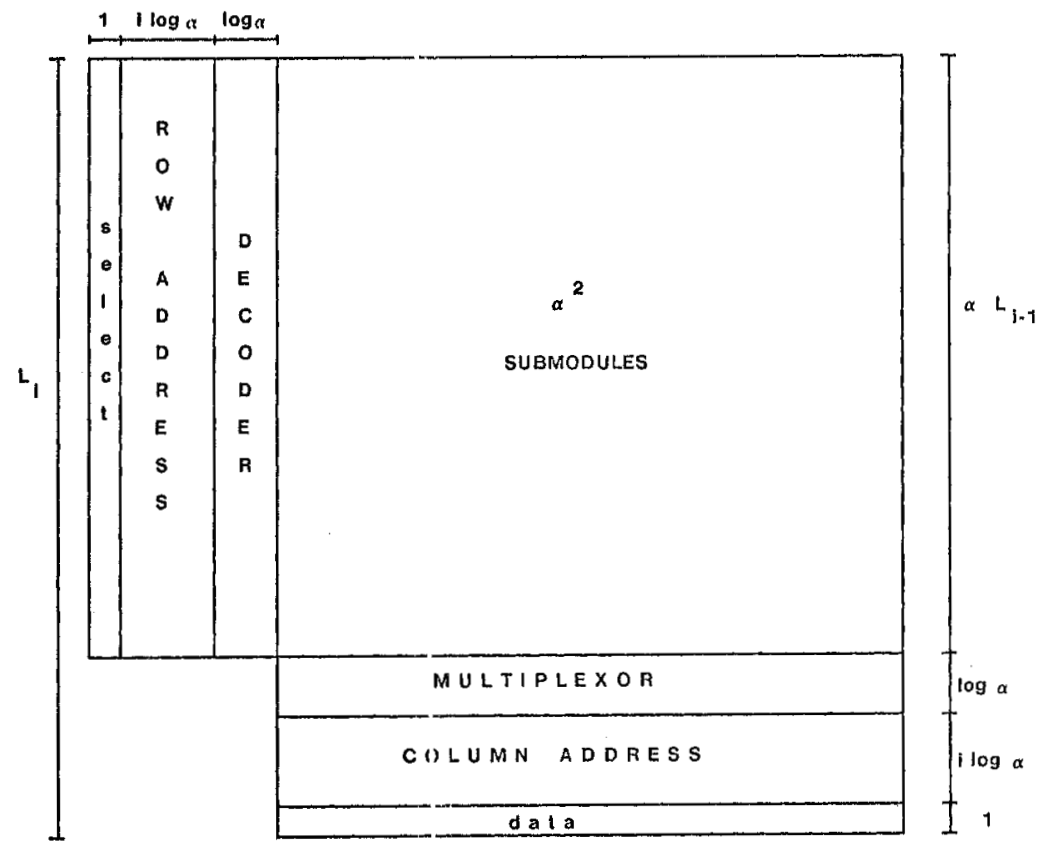

Fig. 4. A RAM module of level $i(i>0)$.

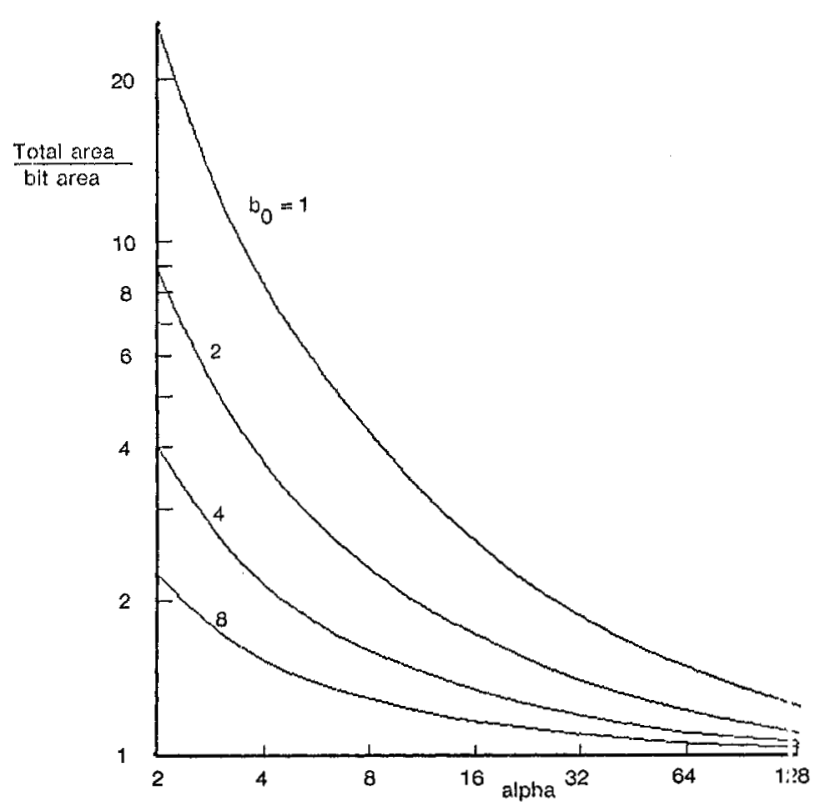

Fig. 5. Total area per bit of a RAM as a function of $\alpha$.

consumed by the wires for power and ground. The reason for this is that these wires can be thought of as increasing only the width $b_{0}$ of each bit; they do this by an amount that is roughly independent of $\alpha$, as is shown in the following analysis.

For simplicity we assume that the wires for power and ground run in opposite directions, say parallel to the data and select wires. We compute how much one of them contributes to the width of a module $i$. The width of a power or ground wire is proportional to the number of bits served by it. Let the wilth at the highest level be $u$; given $S$ and the design of the lowest level memory cell, this parameter is easy to compute. The width of the wire in a module on level $i$ is proportional to the current it must supply and is hence $u\left(\alpha^{2 i} / \alpha^{2 N}\right)$. In one direction, horizontal or vertical, there are $\left(\alpha^{N} / \alpha^{i}\right)$ such modules. The total contribution of all modules on level $i$ is thus $u\left(x^{i}\right)$
$\left.\alpha^{N}\right)$. Taking the sum of this expression for $i=0,1, \cdots, N$ yields

$$
\frac{u}{\alpha^{N}} \frac{\alpha^{N+1}-1}{\alpha-1} \approx u \frac{\alpha}{\alpha-1} .
$$

There are $\sqrt{S}$ bits in one direction; the increase of the bit width, due to power and ground, therefore, is

$$
\frac{u}{\sqrt{S}} \frac{\alpha}{\alpha-1}
$$

which is roughly equal to $u / \sqrt{S}$.

We are interested in the optimal choice of $\alpha$, but to make that choice we will have to look at the access time, which also depends on $\alpha$ as well.

\section{Access Time of the RAM}

Each element of level 0 drives a wire of length $\alpha b_{0}$ to reach the periphery of its module on level 1; this takes time $\alpha b_{0}$. Each module on level 1 drives in the same amount of time as a wire that is $\alpha$ times longer to reach the periphery of its module on level 2, etc. With $N$ being the level of the highest module, the time required to extract 1 bit of storage adds up to $\alpha b_{0} N$. We use this figure as the access time. For a RAM of $S$ words, the access time is then $\alpha b_{0}(\log S / 2 \log \alpha)$.

\section{The Cost of the RAM}

We take the product of the area and the access time as the cost function of the RAM. A RAM of $S$ words of $\log S$ bits each has the following area-time product.

$$
\left(b_{0}+\frac{1}{\alpha-1}+\frac{2 \alpha-1}{(\alpha-1)^{2}} \log \alpha\right)^{2} \frac{\alpha b_{0}}{2 \log \alpha} S \log { }^{2} S .
$$

Fig. 6 shows (5), normalized with respect to $S \log ^{2} S$, as a function of $\alpha$ for different values of $b_{0}$. One notices that for increasing bit sizes the branching ratio of the hierarchy should decrease. Static memories, therefore, should have a smaller $\alpha$ 


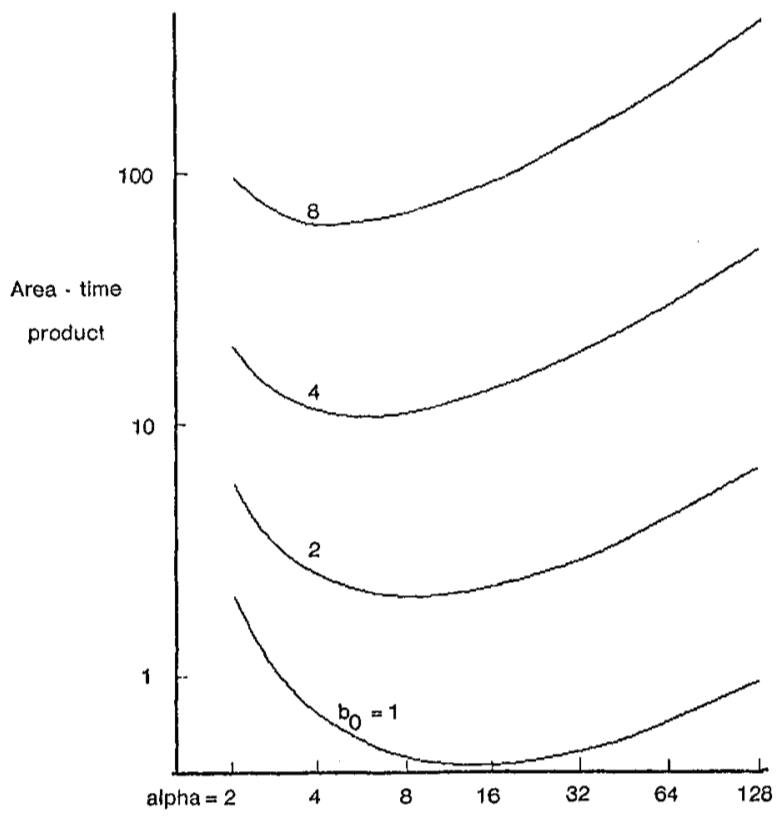

Fig. 6. Area-time product of a RAM as a function of $\alpha$.

than dynamic ones. For dynamic MOS memories the optimal choice for $\alpha$ lies between 8 and 16, for static MOS memories $\left(b_{0} \approx 4\right)$ between 4 and 8 . One may speculate that "smart memories," structures in which part of the processing task is distributed over the memory cells, will have small branching ratios and hence relatively deep hierarchies.

\section{Content Addressable Memory}

The basic elements of the RAM were bits. The content addressable memory (CAM) is an example of a word organized memory. We consider a "pure" CAM. It consists of words of $w$ bits each. We access a word by applying $w$ bits of data to the system. We assume that there is only one word in the memory with that contents, and the address of that word is produced by the memory.

\section{A. Organization of the CAM}

The basic elements are the bits, each of width $b_{1}$. The bits do not constitute the modules of level 0 . The modules on level 0 of the hierarchy consist of $\alpha w$ words of $w$ bits each. [See Fig. 7 (b)] The $w$ data bits are run via parallel wires vertically through the module. Out of each word comes one horizontal match wire going to the right. A word asserts its match wire if each data bit received is equal to the corresponding bit stored. There are $a w$ words in a module of level 0 ; the address of the matching word leaves the module via the $\log \alpha w$ address wires.

The above organization of a module of level 0 has one defect. It would require the individual bits of storage to drive wires of length $w b_{1}$, which may be greater than the desired $\alpha b_{1}$, to reach the address wires. In Section II, we discussed that this type of communication should be achieved by a hierarchy. We, therefore, organize the driving of the match wire by the $w$ bits in a word in the same manner as shown in Section II.

Each word is chopped up into $(w / \alpha)$ subwords of $\alpha$ bits each [Fig. 7(a)]. Each of the $(w / \alpha)$ subwords sends a signal to a "match tree" which has a branching ratio of $\alpha$ and delivers, via $\log _{\alpha} w$ levels, the logical product of its inputs. The top node of the match tree can drive a wire of length $b_{1} \alpha^{\log _{\alpha} w}=$ $b_{1} w$, the length of a word in the memory. Therefore, the word itself can drive a wire of length $b_{1} \alpha w$, and we may group together $\alpha w$ words into module 0 [Fig. 7(b)]. Notice that the module's length is roughly equal to $\alpha$ times its width. This will be true for modules on higher levels as well.

We now describe a module of level $i$ (Fig. 8). It contains $w \alpha^{4 i+1}$ words and consists of $\alpha^{4}$ submodules of level $i-1$, grouped into $\alpha^{2}$ rows of $\alpha^{2}$ submodules each. Each such row contains, besides the $\alpha^{2}$ submodules, $w$ data wires to trans. port the data to each of the submodules and $\log w \alpha^{4 i-1}$ outcoming address wires to transport to the right the address of the matching word. Each submodule has $w \alpha^{4 i-3}$ words, and, hence, one row contains $w \alpha^{4 i-1}$ words which explains the number of address wires. A module on level $i$ has $\alpha^{2}$ of these rows and thus requires $\log w \alpha^{4 i+1}$ outcoming address wires; they are placed to the right of the rows.

In the CAM we have $\alpha^{4}$ submodules per module, in the RAM only $\alpha^{2}$. This is only a seeming difference. In the CAM, for simplicity, we have combined two steps in the hierarchy; we have maintained, however, our multiplication factor $\alpha$ for the wire lengths. $L_{i-1}$, the length of a module of level $i-1$, is roughly equal to $\alpha$ times $W_{i-1}$, the width of a module of level $i-1$. Therefore, module $i-1$ can already drive wires of length $\alpha W_{i-1}$. As a consequence, we can put $\alpha^{2}$ submodules into one row as this would only require the driving of wires of length $\alpha^{2} W_{i-1}$ in each row. But then we can, and this is the second step, combine $\alpha^{2}$ rows as this would require the driving of wires of a length about $\alpha^{2} L_{i-1}$, which is roughly equal to $\alpha^{3} W_{i-1}$.

\section{B. Area of the CAM}

We compute the length and the width separately. For the length $L_{i}$ of a module on level $i$, we have the relation [cf. Figs. $7(\mathrm{~b})$ and 8$]$

$$
\begin{aligned}
& L_{0}=\alpha w\left(b_{1}+\frac{\log w}{\log \alpha}\right) \\
& L_{i}=\alpha^{2}\left(w+L_{i-1}+\log w \alpha^{4 i-1}\right) .
\end{aligned}
$$

The solution to this recurrence relation is

$$
\begin{aligned}
L_{i}= & \alpha^{2 i+1} w\left(b_{1}+\frac{\log w}{\log \alpha}\right)+(w+\log w) \frac{\alpha^{2 i+2}-\alpha^{2}}{\alpha^{2}-1} \\
& +\left(\frac{4 \alpha^{2 i+2}-4 \alpha^{2}}{\left(\alpha^{2}-1\right)^{2}}+\frac{3 \alpha^{2 i+2}-4 i \alpha^{2}-3 \alpha^{2}}{\alpha^{2}-1}\right) \log \alpha .
\end{aligned}
$$

A module on level $i$ has $w \alpha^{2 i+1}$ bits in the vertical direction. The length per bit, therefore, is $L_{i} / w \alpha^{2 i+1}$. This has the following limit for $i \rightarrow \infty$ :

$$
b_{1}+\frac{\log w}{\log \alpha}+\frac{\alpha(w+\log w+3 \log \alpha)}{w\left(\alpha^{2}-1\right)}+\frac{4 \alpha \log \alpha}{w\left(\alpha^{2}-1\right)^{2}} .
$$

As in the case of the RAM, $L_{i} / w \alpha^{2 i+1}$ is already very close to the limit for small values of $i$; the rate of convergence is again exponential. We use (6) as the length per bit of a CAM.

We find for the width $W_{i}$ of a module on level $i$ the following 


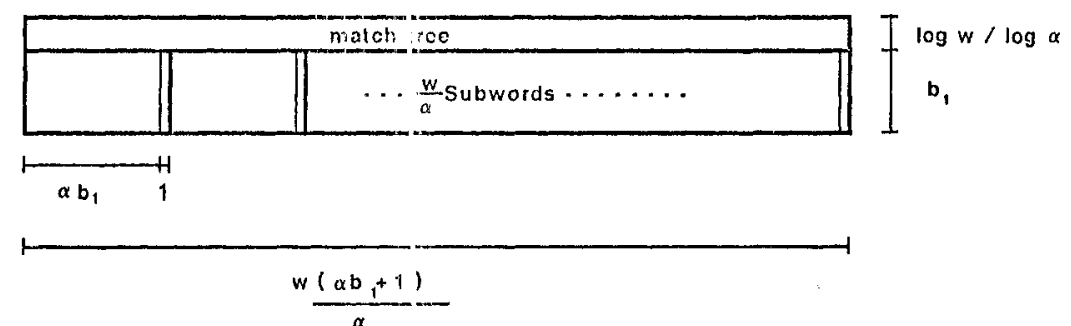

(a)

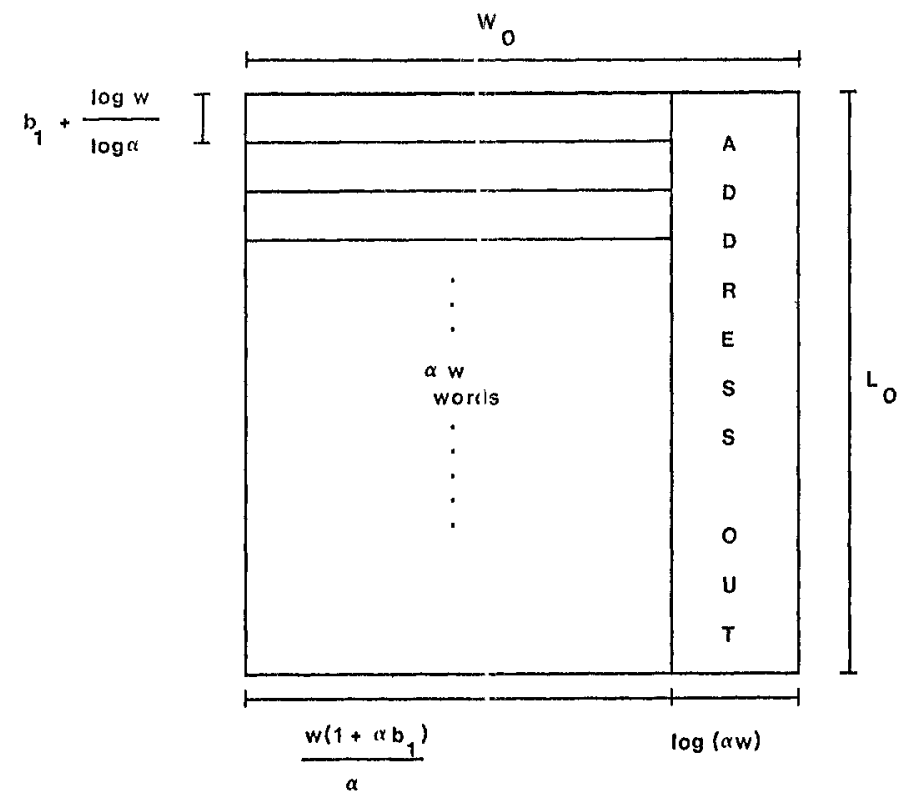

(b)

Fig. 7. (a) One word of storage in the CAM. (b) A CAM module of level zero.

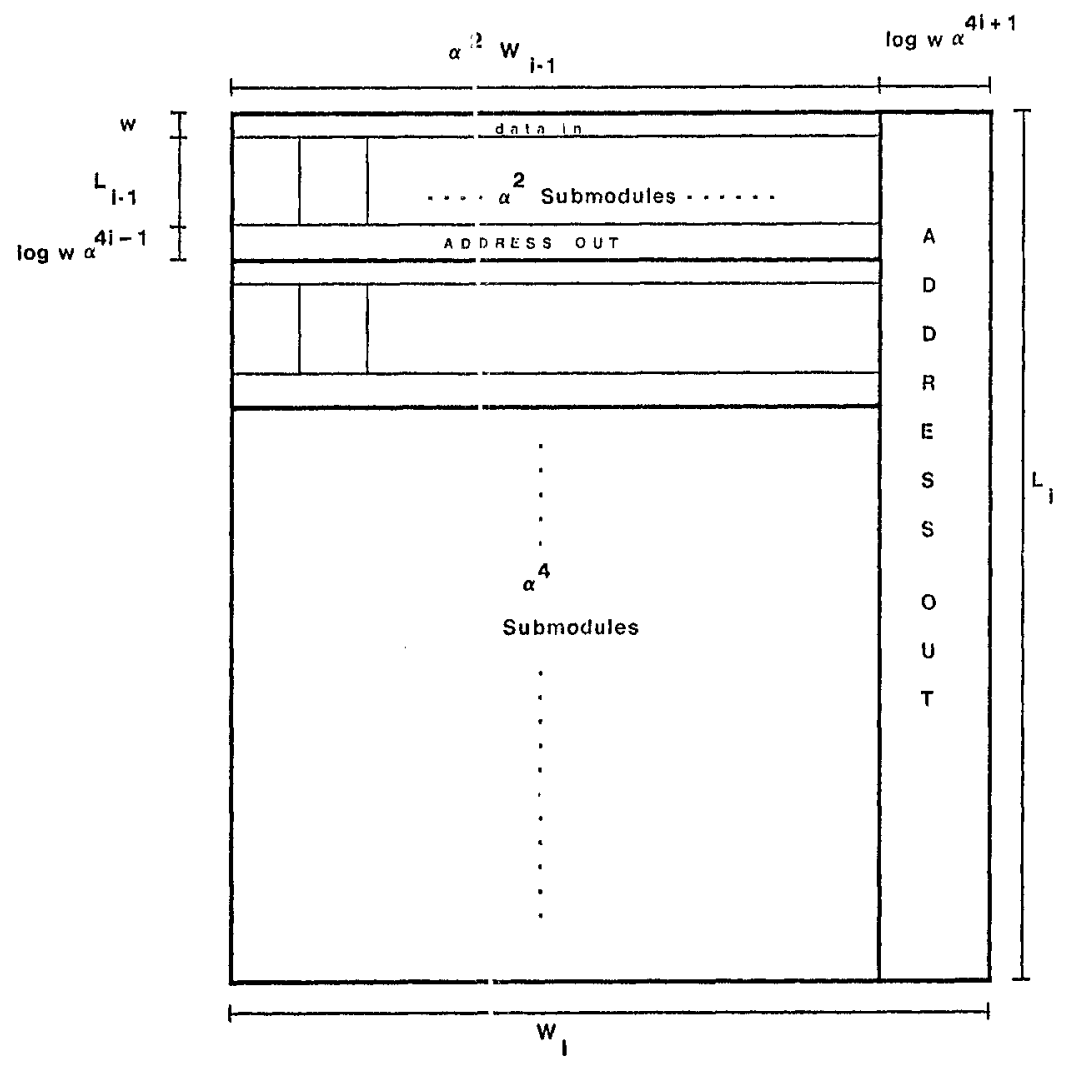

Fig. 8. A CAM module of level $i(i>0)$. 
recurrence relation [cf. Figs. 7(b) and 8]:

$$
\begin{aligned}
& W_{0}=\frac{w}{\alpha}\left(\alpha b_{1}+1\right)+\log \alpha w \\
& W_{i}=\alpha^{2} W_{i-1}+\log w \alpha^{4 i+1} .
\end{aligned}
$$

Its solution is

$$
\begin{aligned}
W_{i}= & \alpha^{2 i} w\left(b_{1}+\frac{1}{\alpha}\right)+\frac{\alpha^{2 i+2}-1}{\alpha^{2}-1} \log w \\
& +\left(\frac{4 \alpha^{2 i+2}-4 \alpha^{2}}{\left(\alpha^{2}-1\right)^{2}}+\frac{\alpha^{2 i+2}-4 i-1}{\alpha^{2}-1}\right) \log \alpha .
\end{aligned}
$$

In the horizontal direction there are $w \alpha^{2 i}$ bits. The width per bit $W_{i} / w \alpha^{2 i}$ has as its limit for $i \rightarrow \infty$

$$
b_{1}+\frac{1}{\alpha}+\frac{\alpha^{2} \log \alpha w}{w\left(\alpha^{2}-1\right)}+\frac{4 \alpha^{2} \log \alpha}{w\left(\alpha^{2}-1\right)^{2}} .
$$

We take the product of $(6)$ and $(7)$ as the area per bit.

By dividing the area per bit by the bit area $b_{1}^{2}$ we obtain the total area per bit area for a CAM. Fig. 9 shows this quotient for $w=32$ as a function of $\alpha$ for different values of $b_{0}$.

If we compare Figs. 5 and 9, we notice that for small values of $\alpha$ the wires in the CAM cause less overhead in area than those in the RAM. For large values of $\alpha$ it is the RAM that enjoys a smaller overhead in area. For equal bit sizes, i.e., with $b_{0}=b_{1}$, the area overhead factor for the RAM and the CAM are about equal at $\alpha=8$.

As in the RAM we can compute by how much we should increase the bit width $b_{1}$ if we wish to take power and ground into account. Both power and ground give an increase of $u\left(\alpha^{2} / \alpha^{2}-1\right)$ to the length and the width of the CAM. This is even closer to $u$ than in the case of the RAM. If we wish to ammortize this amount over the bits, the bit width $b_{1}$ should be incremented by

$$
\frac{2 u}{\sqrt{S w}} \frac{\alpha^{2}}{\alpha^{2}-1}
$$

for a CAM of $S$ words of $w$ bits each.

\section{Access Time of the CAM}

For the access time we take the time required to extract the address of the matching word of data from a memory of $S$ words. With the highest level being level $N$, we have $S=$ $w \alpha^{4 N+1}$ or

$$
N=\frac{\log S-\log w}{4 \log \alpha}-\frac{1}{4} \text {. }
$$

A word of storage has a response time of $(\log w / \log \alpha) \alpha b_{1}$; for a module of level 0 this becomes $[(\log w / \log \alpha)+1] \alpha b_{1}$. Each new level of the hierarchy multiplies the wire lengths by a factor $\alpha^{2}$ and hence requires an additional time of $2 \alpha b_{1}$. For $N$ levels we find, hence,

$$
\begin{aligned}
\text { access time } & =\left(2 N+\frac{\log w}{\log \alpha}+1\right) \alpha b_{1} \\
& =\left(\frac{\log S+\log w}{2 \log \alpha}+\frac{1}{2}\right) \alpha b_{1} .
\end{aligned}
$$

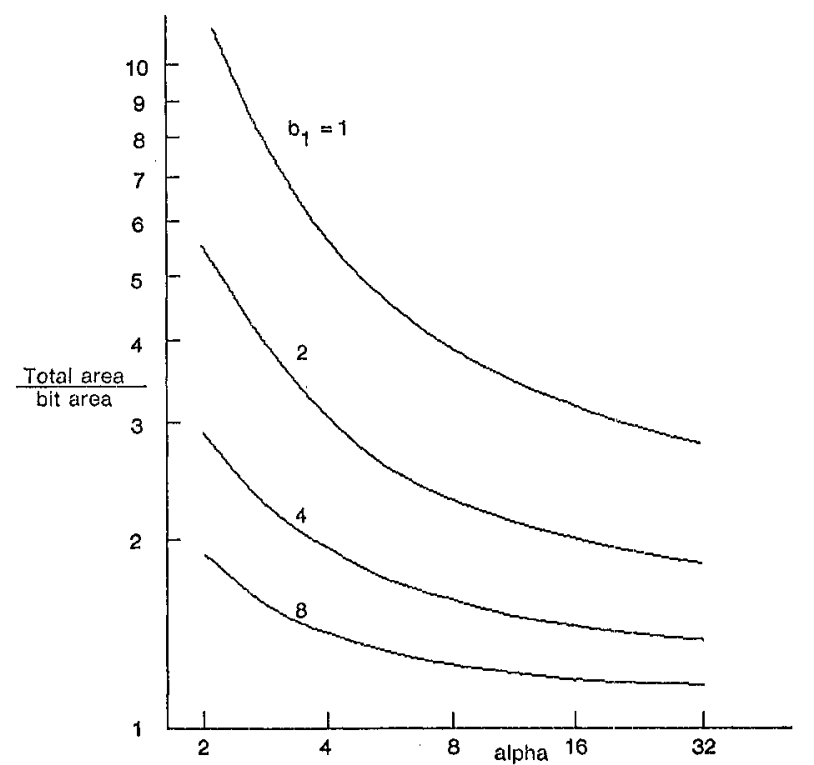

Fig. 9. Total area per bit as a function of $\alpha$ for a CAM with word length 32 .

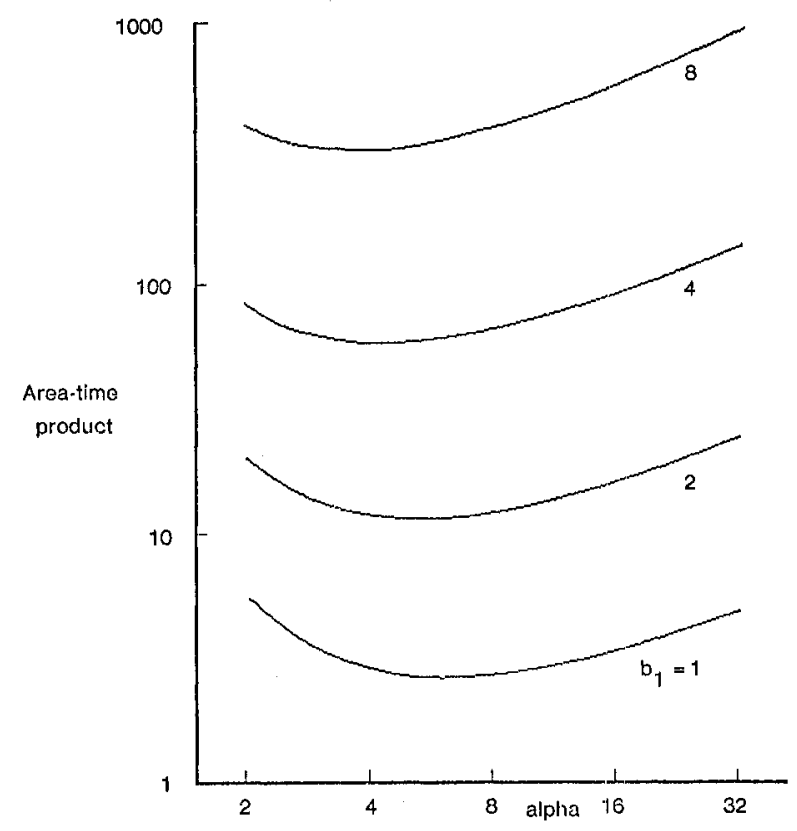

Fig. 10. Area-time product for a CAM of $65 \mathrm{~K} 32$-bit words.

\section{The Cost of the CAM}

We again take the product of the area and the access time as the cost function. For a CAM of $S$ words of $w$ bits each, formulae (6), (7), and (8) yield the cost function

$$
\begin{aligned}
\left(b_{1}+\right. & \left.\frac{\log w}{\log \alpha}+\frac{\alpha(w+\log w+3 \log \alpha)}{w\left(\alpha^{2}-1\right)}+\frac{4 \alpha \log \alpha}{w\left(\alpha^{2}-1\right)^{2}}\right) \\
& \cdot\left(b_{1}+\frac{1}{\alpha}+\frac{\alpha^{2} \log \alpha w}{w\left(\alpha^{2}-1\right)}+\frac{4 \alpha^{2} \log \alpha}{w\left(\alpha^{2}-1\right)^{2}}\right) \\
& \cdot\left(\frac{\log S+\log w}{2 \log \alpha}+\frac{1}{2}\right) \alpha b_{1} w S .
\end{aligned}
$$

Fig. 10 shows the cost function as a function of $\alpha$ for a CAM of $65 \mathrm{~K}$ words of 32 bits each. The curves are fairly indepen- 
dent of the choice of $w$ provided we choose $w$ great enough, say $w \geqslant 16$. A change in $S$ will basically move the curves only up and down; it will not affect the positions of their minina.

We notice again that increasing the bit size will decrease the optimal choice of $\alpha$. Comparing Figs. 6 and 10 we see that content addressable memories should have smaller branihing ratios than random-access memories. For $b_{1}=4$, which seems a reasonable figure, the optimal choice of $\alpha$ is 4 .

\section{CONCLUSION}

We have presented a general method for analyzing the cost and performance of recursively defined VLSI structures. Parameters of any such structure may be optimized with respect to time, area, or some combination of the two. While we have chosen the area-time product, it is clear that some other choice may be appropriate for any given application.

The results of this study indicate that as more processing is available in each module at level zero, the optimal value of $\alpha$ will decrease. A system with $\alpha=4$ would seem to be appropriate for memories in which substantial processing is comirgled with storage.

Very general arguments were used to generate the basic recursive structure. For that reason it appears that a very large fraction of VLSI computing structures will be designed in this way. We have discussed two examples, one in whick the basic elements were bits of storage, and one with worcs of storage at the lowest level. They gave rise to rather different recursive structures. The way in which their area and time measures were established should make it clear how to apply these techniques to other recursively defined computing structures.

Carver A. Mead, for a biography and photograph, see this issue, $p$. 548 .

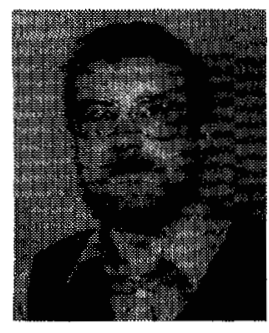

Martin Rem was born in The Netherlands on September 22, 1946. He received the B.S. degree in mathematics and physics and the M.S. degree in mathematics from the University of Amsterdam, Holland, in 1968 and 1971, respectively, and the Ph.D. degree in computer science from Eindhoven University of Technology, Eindhoven, The Netherlands, in 1976.

$\mathrm{He}$ is an Associate Professor of Mathematics in the Department of Mathematics, Eindhoven University of Technology. He is presently a Visiting Professor of Computer Science in the Department of Computer Science, California Institute of Technology, Pasadena, CA. His major research interests are in the area of programming of machines with instore processing, semantics of programming languages, correctness proofs, and well-structured machine designs.

Dr. Rem is a member of the Association for Computing Machinery, the Dutch Computer Society NGI, and the Dutch Mathematical Society.

\title{
Delay-Time Optimization for Driving and Sensing of Signals on High-Capacitance Paths of VLSI Systems
}

\author{
AMR M. MOHSEN, MEMI3ER, IEEE, AND CARVER A. MEAD
}

\begin{abstract}
Transmission of signals on large capacitance paths in a VLSI system may result in substantial degradation of the overall system performance. In this paper minimization of the delay time; associated with driving and sensing signals from large capacitance paths by optimizing the fan-out factor of the driver stages, the gain of the input sensing stages, and the path voltage swing are examined. Examples of driving signals on a high capacitance path with two driving schemes are: a push-pull depletion-load driver chain and a fixed drver; and of sensing signals with two sensing schemes: a single-ended depletion-load inverter input stage and a balanced regenerative strcbed latch are presented. We conclude that minimum delay time is achisved when the delay times of the successive stages of the driver chain, the high capacitance path, and the input sensing stage are comparable.

A. M. Mohsen is with Intel Corporation, Santa Clara, CA, and the California Institute of Technology, Pasadena, CA 91125.

C. A. Mead is with the Department of Computer Science, California Institute of Technology, Pasadena, CA 91125.
\end{abstract}

In general, transmission time of signals in a system is minimized when the delay times of the different stages of the system are comparable.

\section{INTRODUCTION}

$T$ HE OVERALL PERFORMANCE of VLSI systems may be seriously degraded if signals need to be transmitted from one part to other parts in the system across large capacitance paths [1]. This large fan-out situation often occurs in the case of control drivers that are required to drive a large number of inputs to memory cells or logic-function blocks across a chip, or in the case of sensing stored information from small cells of large memory arrays. A similar and even more serious problem is driving wires which go off the silicon chip to other chips or input and output devices. In such cases, the 